\title{
Theoretical analysis of active earth pressure behind a rigid retaining wall
}

\author{
Song Yang ${ }^{1}$, a, Wenlin Geng ${ }^{1}$, Yongfeng Wang ${ }^{1}$, Zhenxing Wang ${ }^{1}$ \\ Yang Liü, ${ }^{2, b}$ and Pengqiang $\mathrm{Yu}^{2}$ \\ ${ }^{1}$ Powerchina Roadbridge Group Co., Ltd., Beijing, China \\ 2 Department of Civil Engineering, University of Science and Technology Beijing 100083, China \\ a93439847@qq.com_byu_pengqiang@163.com
}

\begin{abstract}
Keywords: earth pressure; sandy backfill; two-dimensional differential equilibrium
Abstract. The goal of this study is to precisely estimate the active earth pressure acting on a rigid retaining wall under translating mode. In this study, the problem of the retaining wall with a parabolic slip surface in the backfill behind the wall is investigated using a two-dimensional system of equilibrium, unlike the horizontal flat-element method considering soil arching effect for calculating active earth pressure. New equations are developed to calculate the magnitude and distribution of the active earth pressure.
\end{abstract}

\section{Introduction}

A precise estimation of earth pressure is an extremely essential issue in geotechnical engineering. Conventionally, the classical Rankine and Coulomb theories with a simple consequence of linear distribution have been used to calculate the active earth pressure against rigid retaining walls. However, many experimental studies (Tsagareli [1], Sherif and Fang [2]) show that the distribution of active earth pressure acting on rough walls is nonlinear, and it depends on the mode of wall movement and soil-wall friction angle.

Handy [3] suggested that the nonlinear distribution of active earth pressure acting on a rigid wall should result from soil arching effects. Later, many authors (Paik [4], Geol and Patra [5]) also investigated active earth pressure in terms of soil arching effects by means of minor principal stress trajectory. Paik attempted to apply the arching effects to the horizontal flat-element method in the investigation of active earth pressure against rigid retaining walls. Since then, the method of combining stress trajectory with differential flat elements in a wedge-shaped failure zone to investigate earth pressure attracted many researchers' attention and they also proposed various active earth pressure solutions by different shapes of stress trajectory and the inclined angle of failure surface under a plane slip surface assumption, except the parabolic slip surface used by Geol. (Geol [5], Liu [6], Zhou [7])

In this study, the problem of active earth pressure against a rigid retaining wall under the translation mode is investigated in a two-dimensional equilibrium system. With the assumption of uniform vertical stress in any horizontal plane, theoretical stress solution of any point in the failure zone are derived. Under the assumption of uniform vertical pressure and other reasonable boundary conditions, new solutions of active earth pressure are obtained by solving the two-dimensional equilibrium equations of a differential element.

\section{Proposed the 2D Analytical Method}

Basic Hypotheses. For definiteness and convenience, in this study, the following hypotheses are made:(1) the problem to be studied is plane strain condition. (2) the backfill obeys the Mohr-Coulomb failure criterion; (3) the failure surface is a parabolic surface through the bottom of the wall.

Establish Equilibrium Equations. As illustrated in the Fig. 1, the rectangular coordinate system is placed at the bottom of the retaining wall. For convenience of analysis, the x-axis is measured rightwards horizontally and the z-axis is measured upward vertically. According to the stress state of 
a differential element of the backfill behind the retaining wall in Fig. 1, the differential equilibrium equations can be described as

$$
\begin{array}{r}
\frac{\partial \sigma_{x}}{\partial x}+\frac{\partial \tau_{z x}}{\partial z}=0 \\
\frac{\partial \sigma_{z}}{\partial z}+\frac{\partial \tau_{x z}}{\partial x}=-\gamma
\end{array}
$$

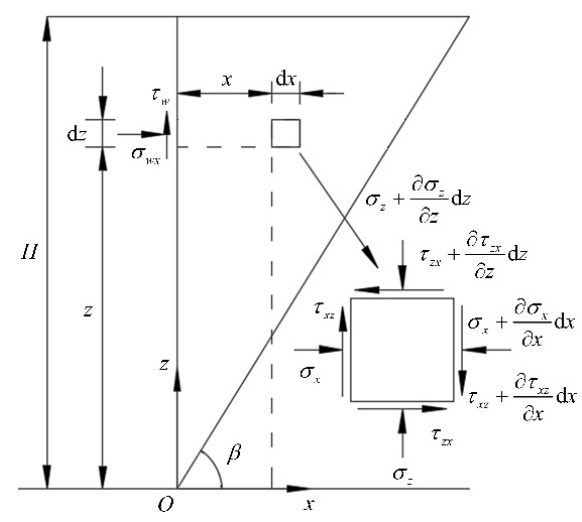

Fig. 1 stress state of a differential element in backfill soil behind a retaining wall

Where $\gamma$ is the unit weight of the backfill which is assumed as a constant value throughout the soil mass. $\sigma_{x}$ and $\sigma_{z}$ are horizontal and vertical stress, respectively; $\tau_{z x}$ is the shear stress along the horizontal direction, and $\tau_{x z}$ is the shear stress along the vertical direction. Note that, the compressive stress is taken as positive.

Boundary Condition The stress state of the backfill adjacent to the retaining wall can be expressed by a constant value $K_{w}$, which can be calculate as follows:

$$
K_{w}=\frac{\sigma_{x w}(z)}{\sigma_{z w}(z)}=\frac{1-\cos (\arcsin (\sin \delta / \sin \varphi)-\delta) \sin \varphi}{1+\cos (\arcsin (\sin \delta / \sin \varphi)-\delta) \sin \varphi}
$$

Where $K_{w}$ is the active lateral stress ratio at the wall, $\sigma_{x w}(z)$ and $\sigma_{z w}(z)$ are the horizontal and vertical stress at the wall, $\delta$ is the wall-soil friction angle, and $\varphi$ is the internal friction angle in which $\delta \leq \varphi$.

The full mobilization of the wall-soil friction angle suggests that shear stress $\tau_{w}$ along the wall can be calculated by Eq.(4):

$$
\tau_{w}(z)=\mu_{w} \sigma_{w x}(z)
$$

Where $\mu_{w}=\tan \delta$.

Further, the shear stress $\tau_{w}$ can be rewritten in the following form by substituting Eq.(3) into Eq.(4):

$$
\left.\tau_{x z}(x, z)\right|_{x=0}=\mu_{w} K_{w} \sigma_{z}(z)=A \sigma_{z}(z)
$$

Where $\left.\tau_{x z}(x, z)\right|_{x=0}=\tau_{w}(z)$, and $A=\mu_{w} K_{w}$ is a defined coefficient for the convenience of writing and derivation.

The shape of emerging failure surface can be defined as a parabolic curve. In this study, the parabolic equation is defined in Eq.(6):

$$
z=\left[\left(\tan ^{2} \alpha-\tan ^{2} \beta\right) / 4 H\right] x^{2}+x \tan \beta
$$

Where $\alpha=\pi / 4+\varphi / 2, \beta=\arctan \left(\sqrt{\tan ^{2} \varphi+\frac{\tan \varphi}{\tan (\varphi+\delta)}}+\tan \varphi\right)$

The stress ratio $B=\tau_{x z}(x, z) / \sigma_{z}(z)$ of the soil in the failure surface is assumed to be identical to that obtained from the Mohr circle at the state of failure, so we can get: 


$$
B=\left.\frac{\tau_{x z}}{\sigma_{z}}\right|_{x=\left(\sqrt{b^{2}+4 a z}-b\right) / 2 a}=\frac{\sin \varphi \sin 2\left(\pi / 2+\varphi-2 \arctan \left(\sqrt{b^{2}+4 a z}\right)\right)}{1+\sin \varphi \cos 2\left(\pi / 2+\varphi-2 \arctan \left(\sqrt{b^{2}+4 a z}\right)\right)}
$$

Stress Solutions. Due to the assumption on uniformly distributed $\sigma_{z}$ at arbitrary depth, we can get Eq.(8) after a partial differentiation with respect to $\mathrm{x}$ taken to Eq.(2):

$$
\tau_{x z}(x, z)=C_{1}(z) x+C_{2}(z)
$$

With the Eq. 5 and Eq.7, substitution of Eq.(8) into Eq.(2) obtains the following differential equation:

$$
\frac{\mathrm{d} \sigma_{z}}{\mathrm{~d} z}+\frac{2 a(A-B)}{b-\sqrt{b^{2}+4 a z}} \sigma_{z}=-\gamma
$$

It is convenient to get a numerical solution of $\sigma_{z}$ by solving Eq.(9) using Matlab software. The active horizontal earth pressure $\sigma_{w x}$ is equal to $\sigma_{x}(x, z)$ at $\mathrm{x}=0$ for a certain depth $\mathrm{z}$, so $\sigma_{w x}$ can be calculated by the following equation:

$$
\sigma_{w x}(z)=\left.\sigma_{x}(x, z)\right|_{x=0}=K_{w} \sigma_{z}
$$

The horizontal active force $P_{a h}$ acting on the wall can be obtained as follows by integrating Eq.(10) with respect to $\mathrm{z}$ :

$$
P_{a h}=\int_{0}^{H} \sigma_{w x} \mathrm{~d} z
$$

From the previous analysis that soil-wall friction angle is fully mobilized, it is accepted that the total active force acting on the rigid wall $P_{a}$ intersects the normal to the wall with an angle $\delta$ and can be expressed as follows:

$$
P_{a}=\frac{P_{a h}}{\cos \delta}
$$

\section{Parametric Analysis}

Fig. 2 shows the variation of the lateral active earth pressure $\sigma_{w x}$,normalized by $\gamma H$, on a $4 \mathrm{~m}$ high, translating wall with $\delta=\varphi$ obtained from the proposed formulations as a function of the internal friction angle. It can be found from the figure that with the increase of internal friction angle from 0 , the distribution of the lateral active earth pressure changes from triangular to nonlinear. Besides, as the soil friction angle increases, the lateral active earth pressure exerting on the retaining wall decreases at each depth and the height of the maximum of earth pressure strength increases.
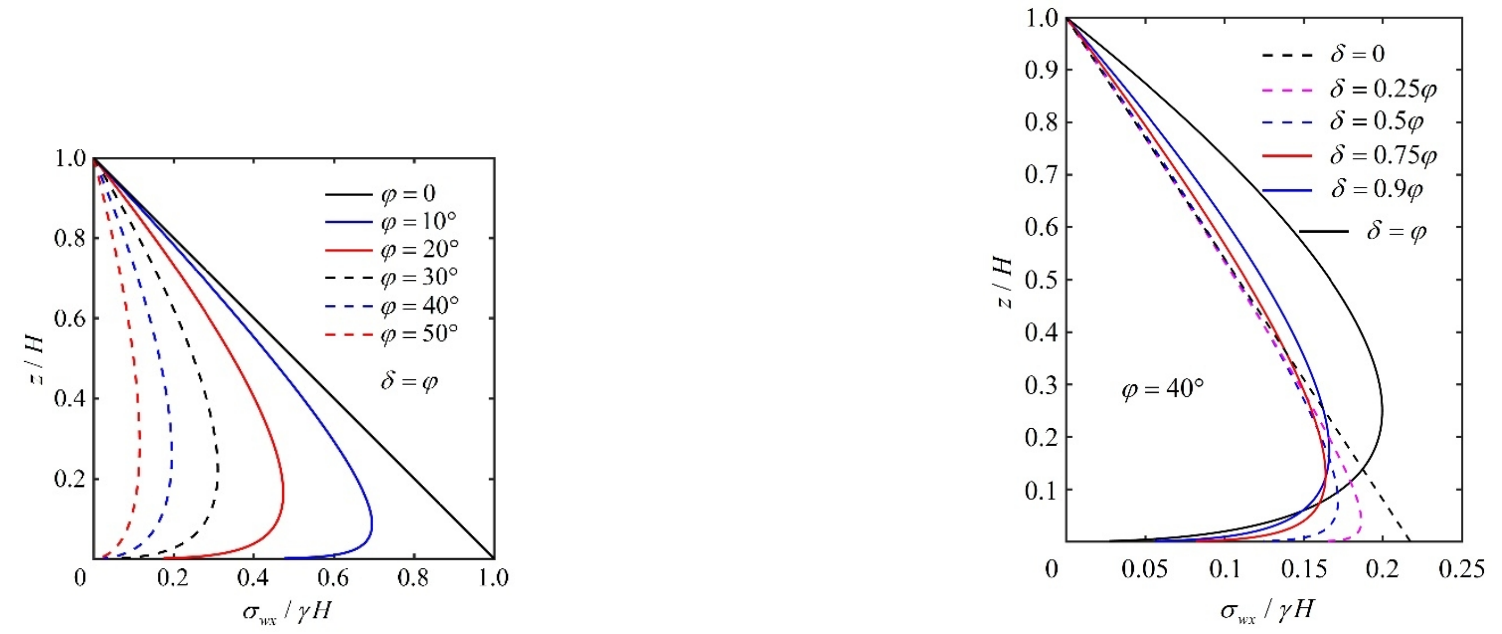

Fig. 2 Change of active earth pressure distribution with $\varphi$

Fig. 3 Change of active earth pressure distribution with $\delta$ 
The distribution of lateral active earth pressure along the wall, normalized by $\gamma H$, in terms of the soil-wall friction angle for $\varphi=40^{\circ}$ is shown in Fig. 3. It is obvious from this figure that the distribution of the horizontal active earth pressure for $\delta=0$ is triangular, which coincides with Rankine theory, and it changes from triangular into nonlinear with the increasing soil-wall friction angle $\delta$. In addition, it is seen from Fig. 3 that with the soil-wall friction angle increasing, the lateral active earth pressure in the upper zone of the wall gradually reduces, while that close to the bottom of the wall becomes larger.

\section{Conclusions}

The problem of rigid vertical retaining walls under translation mode has been studied with a two-dimensional equilibrium system, without a previous assumption of the shape of soil arch. The emerging failure surface was defined using a parabolic equation. Consequently, the numerical solutions of active earth pressure were obtained with the assumption of a uniformly distributed $\sigma_{z}$ at a horizontal height. The distribution of the lateral active earth pressure changes from triangular to nonlinear with the increase of internal friction angle from 0 and the height of the centroid of the earth pressure distribution increases.

\section{References}

[1] Z.V. Tsagareli Experimental investigation of the pressures of a loose medium on retaining walls with a vertical back face and horizontal backfill surface[J]. Soil Mechanics and Foundation Engineering, 1965, 2(4): 197-200.

[2] Sherif M A, Fang Y S, Sherif R I. $K_{a}$ and $K_{0}$ behind rotating and nonyielding walls[J]. Journal of Geotechnical Engineering, 1984, 110(1): 41-56.

[3] HANDY R L. The arch in soil arching[J]. Journal of Geotechnical Engineering, 1985, 111(3): 302-318.

[4] PAIK K H, SALGADO R. Estimation of active earth pressure against rigid retaining walls considering arching effects[J]. Geotechnique, 2003, 53(7): 643-653.

[5] GEOL S, PATRA N R. Effect of arching on active earth pressure for rigid retaining walls considering translation mode[J]. International Journal of Geomechanics, 2008, 8(2): 123-133.

[6] LIU Zhong-yu, CHEN Jie, LI Dong-yang. Calculation of active earth pressure against rigid retaining wall considering shear stress[J]. Rock and Soil Mechanics, 2016, 37(9): 2443-2450. (in Chinese)

[7] Yi-tao Zhou, Qing-sheng Chen, Fu-quan Chen, Xiao-hui Xue, Sudip Basack. Active earth pressure on translating rigid retaining structures considering soil arching effect[J]. European Journal of Environmental and Civil Engineering, 2016, 1-17. 